\title{
Spontaneous Rupture of Subserous Uterine Vein in Late Pregnancy
}

\author{
F YASMIN $^{1}$, N KABIR $^{2}$, M FERDOUS $^{3}$
}

\begin{abstract}
:
Objective: Premature uterine contraction caused by spontaneous rupture of subserous uterine vein resulting intrauterine asphyxia.

Method \& Results: A primigravida with uneventful pregnancy having regular antenatal care attended a private clinic at her 38 week of pregnancy with slight pain in whole abdomen and hardening of uterus. After giving rest in left lateral position \& oxygen inhalation, hardening of uterus persisted. By that time she developed fetal tachycardia and had to undergo caesarean section, there was hemoperitonum and an asphyxiated male baby was delivered. On exploration a subserous uterine vein was detected on the posterior wall of the uterus. Complete hemostasis was achieved with interrupted sutures and electrocauterization of the bleeding points. Post-operative period was uneventful for the mother but the baby was managed in neonatal care unit. Both of them were well during discharge.

Conclusion: Monitoring of pregnant women at last trimester is very important. Any deviation from normal like hardening of uterus should be carefully taken care of for good maternal and fetal outcome.
\end{abstract}

\section{Introduction:}

Hemoperitoneum or spontaneous abdominal hemorrhage is defind as the presence of blood in the peritoneal cavity from a nontraumatic and noniatrogenic cause. Common source of spontaneous abdominal hemorrhage are visceral (hepatic,splenic,renal, adrenal), gynecologic and obstetric, coagulopathy-related, and vascular ${ }^{1,2}$. The common gynecologic causes of hemoperitoneum in pregnancy are rupture of an ectopic pregnancy or rupture of an ovarian cyst, and uterine fibroid, lesion resulting from pelvic endometrial implants and chronic inflammatory disease ${ }^{3}$. Obstetric causes of hemoperitoneum are HELLP syndrome, placenta increta, ruptured uterus. The clinical presentation is usually nonspecific ; thus , frequently the diagnosis is delayed or missed. . Hemoperitoneum is generally classified as a surgical emergency; in most cases, urgent laparotomy is needed to identify and control the source of the bleeding ${ }^{4}$.In selected cases, careful observation may be permissible. The abdominal cavity is highly distensible and may easily hold greater than five liters of blood. Therefore, rapid blood loss into the abdomen may induce hemorrhagic shock and if untreated, rapidly may lead to death ${ }^{4}$. The rarity of spontaneous hemoperitoneum in pregnancy lead to misdiagnosis of the condition, so high index of suspicion is warranted.

\section{Case Report:}

A primigravida of $26 y$ rs was admitted in a private clinic near Dhaka on December 2011 at her 38 weeks of pregnancy with abdominal pain and hardening of abdomen. She was married for 3years and had history of painful menstruation. She conceived spontaneously after trying for one year

1. Dr. Farida Yasmin, Associate Professor, Department of Obstetrics and Gynaecology, Institute of Child and Mother Health, Dhaka.

2. Dr. Nazneen Kabir, Professor, Department of Obstetrics and Gynaecology, Institute of Child and Mother Health, Dhaka.

3. Dr. Menoka Ferdous, Assistant Professor, Department of Obstetrics and Gynaecology , ShaheedShuhrawardyMedicalCollege,Dhaka. 
and was under regular antenatal care. In her late pregnancy she used to feel slight pain in abdomen off and on. But physical examination and laboratory investigations revealed no abnormality. On admission her pulse rate was rapid, but blood pressure was normal. Physical examination showed contracted uterus, fetal tachycardia, closed cervix and there was no abnormal vaginal discharge. Emergency caesarean section was undertaken for persistant uterine contraction and fetal tachycardia. Surprisingly there was free blood in the peritoneal cavity. Surface vessels over the anterior wall of uterus was visible and intact. An asphyxiated male baby weighing $2.5 \mathrm{~kg}$ was delivered and admitted in neonatal care unit for further care. During caesarean section there was no sign of retro placental bleeding, tear or trauma on the uterus. After closing the uterus abdominal cavity was explored thoroughly, a bleeding vein was detected on posterior surface of uterus. Complete hemostasis was achieved with interrupted sutures and electrocauterization of the bleeding points. Abdomen was closed with a peritoneal drain in situ. The patient received one unit of whole fresh blood peroperatively. Her postoperative period was uneventful and she was discharged with her newborn on the $6^{\text {th }}$ postoperative day.

\section{Discussion:}

Spontaneous hemoperitoneum during pregnancy is a very rare, life threatening condition, associated with high fetal and maternal mortality and difficult to diagnose. The blood flow in the pregnant uterus is very high. This high rate of flow emphasizes the possible fatal consequences of delayed therapeutic intervention ${ }^{2}$. The precise causes and incidence of hemoperitoneum during pregnancy are still unknown 5 .

Spontaneous hemoperitoneum is a potentially lifethreatening entity with an extensive differential diagnosis. Gynecologic, splenic, and hepatic etiologies are the most common ${ }^{6,7}$.

Important gynecologic causes of spontaneous hemoperitoneum include ruptured ectopic pregnancy, ruptured ovarian cyst, and endometriosis ${ }^{6,7}$. Rare gynecologic causes of spontaneous hemoperitoneum include various benign and malignant neoplasms ${ }^{8}$,hemorrhagic corpus luteum cyst torsion 7 , and spontaneous uterine rupture in the first trimester of pregnancy ${ }^{9}$.The differential diagnosis of intraperitoneal pain and /or hemorrhage in the third trimester of pregnancy are abruptio placenta, uterine rupture, placenta increta ${ }^{10}$ billiary colic , haemorrhagic pancreatitis, rupture of extrauterine pregnancy,twisted ovarian cyst, renal colic, degeneration of uterine fibroid ${ }^{1}$. Reported cause of spontaneous intra abdominal haemorrhage in the second half of pregnancy was endometriosis ${ }^{11}$. One study by Zhang Y, Zhao Y, in 2009 showed spontaneous intra-abdominal bleeding in the third trimester of pregnancy due to a lesion resulting from endometriosis and chronic pelvic inflammatory disease ${ }^{12}$. Other reported causes were placenta percreta $^{13}$, spontaneous rupture of uterine vessels ${ }^{14,15}$, spontaneous rupture of varicose veins on the surface of the uterus ${ }^{16}$ spontaneous rupture of a previously unknown scarred uterus ${ }^{17}$ or could be idiopathic ${ }^{18}$.

Retroperitoneal bleeding leads to intraperitoneal bleeding, bleeding from utero-ovarian veins according to Ziereisen $\mathrm{V}$ et al. due to spontaneous rupture of utero-ovarian vessels in postpartal period ${ }^{19}$. He also stated that when the event occur with labor maternal mortality is very high,(40\%),than occuring outside of labor $^{20}$, ruptured broad ligament hematoma, ruptured aortic aneurysms or aneurysms of the splenic, renal, iliac, and haemorroidal veins may be the cause of hemoperitoneum. One might speculate that homodynamic stress during pregnancy or labor may put pressure on already previously quiescent aneurismal or malformed blood vessels leading to acute hemorrhage ${ }^{3,21}$. Spontaneous renal or perirenal hemorrhage may also result from coagulopathy, vasculitis and erosion of a vessel by an neoplastic or inflammatory disorder ${ }^{3,22}$.

In the present case maternal general condition was not so deteriorated but there was fetal tachycardia. Emergency caesarean was planned and performed without confirming the definite diagnosis. CT is the single most important imaging technique in the detection and characterization of spontaneous intraperitoneal bleeding 23 .

A review of all cases of spontaneous hemorrhage in pregnancy over the past 20 years showed that $80 \%$ were of venous origin , 16\% arterial origin, and 4\% unknown. The bleeding site in $90 \%$ of cases was either the posterior side of the uterus or the parametrium ${ }^{24}$.

More recent data have shown a dramatic decline in mortality, this change is secondary to advances in resuscitative efforts and anesthesia techniques ${ }^{19,20}$.

\section{Conclusion:}

Spontaneous rupture of utero-ovarian vessels in 
pregnancy can often be misdiagnosed. Obstetricians should be aware of this rare etiology of hemoperitoneum. Studies have shown that timely diagnosis can prevent adverse pregnancy outcome. So the condition merits serious consideration in differential diagnosis of many emergencies associated with pregnancy.

\section{References:}

1. Furlan A, Fakhran S, Michael P F. Spontaneous Abdominal Haemorrhage: Causes,CT. Findings and clinical Implications. American Journal of Roentgenology. 2009;193:1077-1087.

2. HannaW.A. T.J.M.Myles. Spontaneous Intraperitoneal Haemorrhage During Pregnancy: Report of Three Cases. BMJ.1964; 1: 10241026.

3. Munir SI, Tammy L, Seaton J. Spontaneous rupture of utero-ovarian vessels in pregnancy. BMJ case Reports 2012; doi.1136/bcr. 02.2012 .5904

4. Wikipedia, the free encyclopedia. February 2013

5. KimT. H, LeeH. H. Case report Hemoperitoneum during pregnancy with endometriosisreport of four cases.Iranian Journal of Reproductive Medicine. 2010;.8(2):90-93.

6. Lucey BC, Varghese JC, Anderson SW, Soto JA. Spontaneous hemoperitoneum: a bloody mess. Emerg Radiol. 2007;14(2):65-75.

7. Lucey BC, Varghese JC, Soto JA. Spontaneous hemoperitoneum: causes and significance. Curr Probl Diagn Radiol. 2005;34(5):182-195. .

8. Elbiss HM, Neale E. Uterine leiomyosarcoma mimicking a ruptured aortic aneurysm.J Obstet Gynaecol. 2006;26(1):85-86.

9. Park YJ, Ryu KY, Lee JI, Park MI. Spontaneous uterine rupture in the first trimester: a case report. Journal of Korean Medical.Science. 2005;20(6):1079-1081.

10. Ikedilo A, Bibum G. Spontaneous Uterine Artery -Rupture in a Third- Trimester Pregnancy. The Female Patient. 2011;36(11): 39-42.

11. Passos F, Calhaz-Jorge C, Graca LM. Endometriosis is a possible risk factor for spontaneous hemoperitoneum in the third trimester of pregnancy. Fertil Steril. 2008;89(1):251-252.

12. Zhang $Y$, Zhao $Y$, Wei $Y$, Li R, Qiao J. Spontaneous rupture veins during late pregnancy after in vitro fertilization. Fertil Steril.2009 Jul; 92(1):395

13. Roca LE, II, Hoffman MC, Gaitan LF, Burkett G. Placenta percreta masquerading as an acute abdomen. Obstet Gynecol. 2009;113(2Pt 2):512-514.

14. Aziz U, Kulkarni A, Lazic D, Cullimore JE. Spontaneous rupture of the uterine Vessels in pregnancy. Obstet Gynecol. 2004;103(5, Pt 2):1089-1091.

15. Kalaichandran S. Spontaneous haemoperitoneum in labour from ruptured utero-ovarian vessels. J R Soc Med 1991;84:372-373.

16. Hashimoto K, Tabata C, Ueno Y, Fukuda H, Shimoya K, Murata Y. Spontaneous rupture of uterine surface varicose veins in pregnancy: a case report. J Reprod Med. 2005;51(9): 722-724.

17. Nkwabong E, Kouam L, Takang W. Spontaneous uterine rupture during pregnancy: Case report and review of literature. Afr J Reprod Health. 2007;11(2):98-103.

18. Koifman A, Weintraub AY, Segal D. Idiopathic spontaneous haemoperitoneum during pregnancy. Arch Gynecol Obstet. 2007; 276: 269-270.

19. Ziereisen V, Bellens B,Gerard C, Baeyen L. Spontaneous rupture of utero-ovarian vessels in post partal period: a case report and review of the literature. J Gynecol obstet Biol Reprod (paris). 2003; 32(1): 51-4.

20. Monera A, Reynolds A, Baptista P, Costa AZ, Bernardes J. Case report : intrapartum uteroovarian vessels rupture. Arch Gynec obst 2009; 279 (4): 583-5.

21. Khairun Nahar, Hamida Begum, Mahmuda Khatun. Spontaneous Hemoperitoneum from a Ruptured Superficial Uterine Vessel in Third Trimester of Pregnancy. Bangladesh J Obstet Gynaecol. 2008; 23(1): 32-34.

22. Becker R, Kowalsky BL, Hatzmann W. Rupture of the uterine artery after spontaneous delivery with unusual course in childbed. Z Geburtshilfe Neonatal.2002; 206(3): 107-

23. Mortele KJ, Cantisani V, Brown DL, Ros PRSpontaneousintraperitoneal hemorrhage: imaging features.Radiol Clin North Am. 2003; 41(6):1183-201.

24. Brosens IA, Fusi L, Brosens JJ. Endometriosis 